\title{
A hypothesis for the alternative southern branch of the North Anatolian Fault Zone, Northwest Turkey
}

KB Türkiye'de Kuzey Anadolu Fay Zonu'nun alternatif Güney Kol'u için bir hipotez

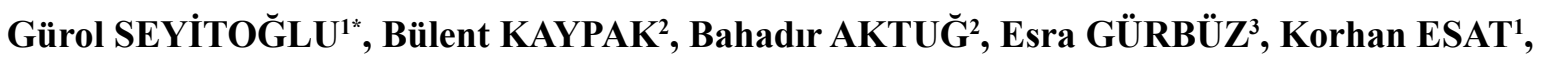 Alper GÜRBÜZ ${ }^{4}$}

\author{
${ }^{1}$ Ankara Üniversitesi, Jeoloji Müh. Bl., Tektonik Araştırma Grubu, Tandoğan, Ankara \\ (e-mail:seyitoglu@ankara.edu.tr) \\ ${ }^{2}$ Ankara Üniversitesi, Jeofizik Müh. Bl., Gölbaşı, Ankara \\ ${ }^{3}$ Aksaray Üniversitesi, Jeoloji Müh. Bl., Aksaray \\ ${ }^{4}$ Niğgde Üniversitesi, Jeoloji Müh. Bl., Niğde \\ * Corresponding author: Tel: 90312203 3370, Fax: 903122150487
}

\begin{abstract}
This paper proposes an alternative route for the southern branch of the North Anatolian Fault Zone (NAFZ) using evidence from morphotectonic features, seismology, GPS and recently published Magnetotelluric and Transient Electromagnetic (MT) data. In this new route, the southern branch connects with the main branch of the NAFZ in Bolu via the Gölpazarı pull-apart basin and Mudurnu. The slip distribution of the NAFZ as taken from GPS data indicates that the newly hypothesized route is the second most important branch of the NAFZ.
\end{abstract}

Keywords: Earthquake, GPS, Neotectonics, North Anatolian Fault Zone, Turkey

\section{$\ddot{O} Z$}

Bu makale morfotektonik özellikleri, sismoloji, GPS ve MT verilerini kullanarak Kuzey Anadolu Fay Zonu'nun (KAFZ) güney kol'u için alternatif bir güzergah önermektedir. Bu yeni güzergahta güney kol, KAFZ'nun ana koluna Gölpazarı çek-ayır havzası ve Mudurnu üzerinden geçerek Bolu'da bağlanmaktadır. GPS verilerinden hesaplanan KAFZ'nundaki kayma dağllımı, yeni önerilen güzergahın KAFZ'nun Marmara'daki ikinci önemli kolu olduğunu göstermektedir.

Anahtar Kelimeler: Deprem, GPS, neotektonik, Kuzey Anadolu Fay Zonu, Türkiye 


\section{INTRODUCTION}

The North Anatolian Fault Zone (NAFZ) is composed of a series of right lateral strike-slip segments (Ketin, 1948) extending from eastern Anatolia to the northern Aegean Sea and it constitutes one of the main neotectonic elements in the eastern Mediterranean (McKenzie, 1972) (Figure 1a). The NAFZ has been active since the Late Miocene time and has an estimated offset of 25-85 km (Seymen, 1975; Şengör, 1979; Barka, 1992). This long term slip rate appears to agree with the current slip rate measured as $17-26 \mathrm{~mm} / \mathrm{yr}$ by GPS data (Barka and Reilinger, 1997; Reilinger et al., 2006). The middle and easternmost parts of North Anatolian Fault Zone have been studied separately with GPS measurements, middle part (Yavaşoğlu et al., 2011), eastern part (Tatar et al., 2012) and Yedisu segment (Özener et al., 2010; Aktuğ et al., 2013a). Very recently, a complete GPS analysis of both the middle and easternmost parts of North Anatolian Fault Zone, including the continuous GPS stations, was given in (Aktuğ et al., 2015). The latter particularly deserves attention since it incorporates all the available GPS data used in earlier studies and presents a comparative analysis of GPS, inSAR and geological estimates in the literatüre.

The eastern sector of the NAFZ is limited to a narrow zone, but widens to the west (Şengör et al., 2005) where shear is distributed along three major branches (Figure 1b). The northern branch passes through Lake Sapanca, the İzmit Gulf, the Marmara Sea and the Saros Gulf. The middle branch is separated from the Almacik flake and passes through Geyve - Pamukova, Lake İznik, and the Gemlik Gulf before turning southwest in the Bandirma Gulf. The northeast end of the southern branch has previously been thought to diverge from the middle branch via Mekece before continuing southwest through Yenişehir, Bursa, south of Lake Ulubat, Mustafakemalpaşa and eventually turning southwest around Gönen (Figure 1b) (Şengör, 1979; Barka and KadinskyCade, 1988; Koçyiğit, 1988; Barka and Kuşçu, 1996; Emre et al., 1997; Armijo et al., 1999; 2002; Yaltırak, 2002; Gürer et al., 2003; 2006; Şengör et al., 2005; Kurtuluş and Canbay, 2007; Yılmaz and Koral, 2007; Selim and Tüysüz, 2013; Le Pichon et al., 2014).

A recent active fault map of Turkey, however, considers that the southern branch is connected to the Eskişehir Fault Zone via active normal faulting between Bursa and İnegöl (Emre et al., 2011).

In contrast, the recognition of the Gölpazarı basin as a pull-apart system (see below), and recently published MT data (Figure 1c) (Kaya, 2010) suggest that the southern branch may extend east through the Gölpazarı pull-apart basin.

Here, we review the morphotectonic, seismic and MT data and propose a hypothesized new route for the southern branch (Figures 1 and 2a), ultimately representing a nearly $100 \mathrm{~km}$ shift of the southern branch of the NAFZ. 


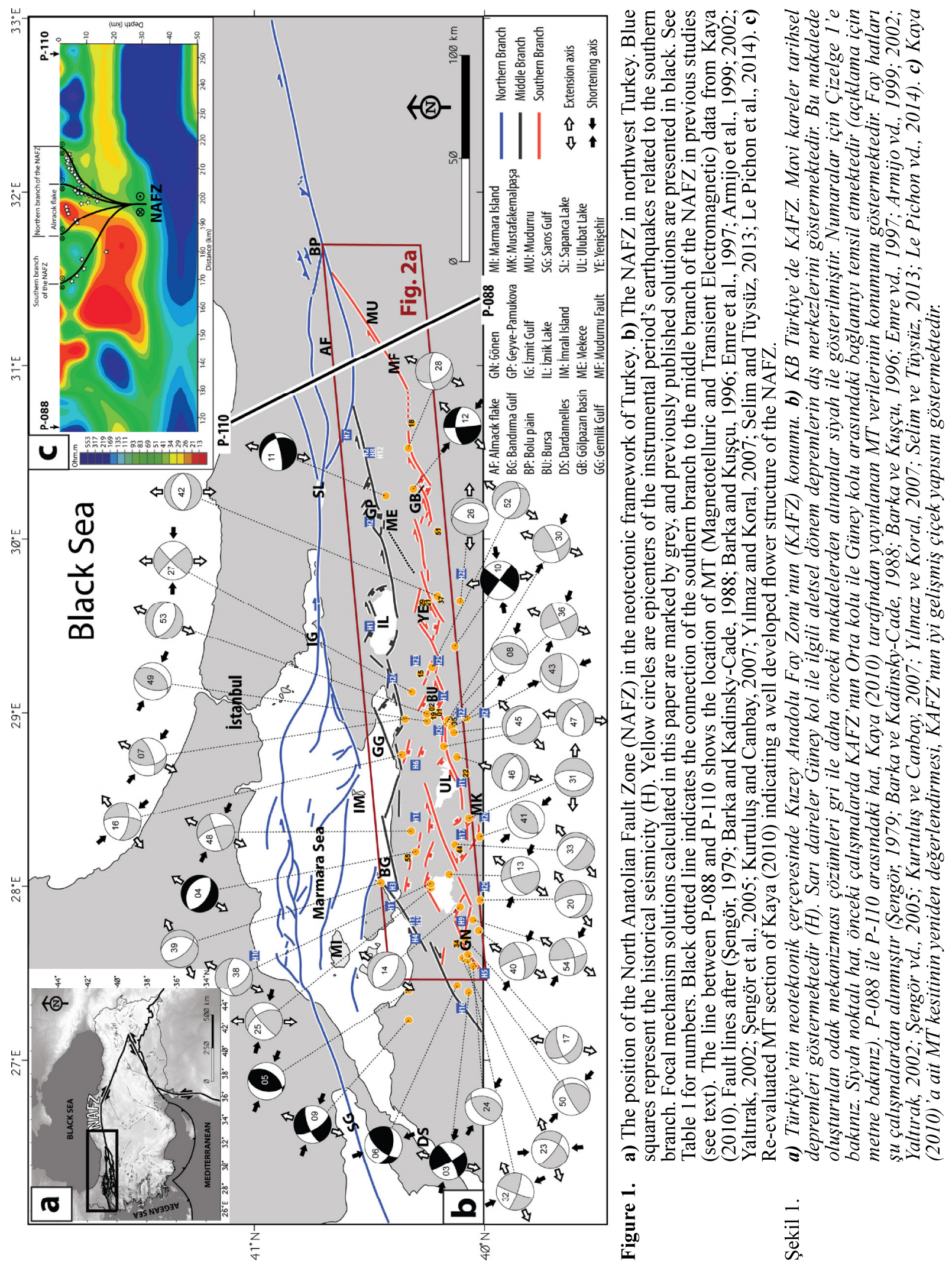




\section{GÖLPAZARI PULL-APART BASIN}

The Gölpazarı basin has previously been evaluated as a karstic depression (Ekmekçi and Nazik, 2004) and/or a graben (Çağlar and İşseven, 2004). However, recent publications (Önde and Gürbüz, 2010; Gürbüz and Seyitoğlu, 2014) describe Gölpazarı basin as a $12 \mathrm{~km}$ by $4.25 \mathrm{~km}$ ideal rhomboidal shaped pull-apart structure. The east northeast - west southwest trending right lateral strike-slip faults create a releasing offset that limits the northwest and southeast margins of the basin, whereas the northeast and southwest margins are bounded by normal faults (Figure $2 b$ ). The basin contains only Quaternary deposits $\geq 300 \mathrm{~m}$ thick, as reported by the State Hydraulic Works (Gürbüz and Seyitoğlu, 2014). The Gölpazarı pull-apart basin is the small-scale equivalent of the adjacent Yenişehir pull-apart system (Y1lmaz and Koral, 2007; Gürbüz, 2010), suggesting it is located on the newly hypothesized route of the southern branch of the NAFZ as described in this paper.

\section{THE ALTERNATIVE ROUTE OF THE SOUTHERN BRANCH}

The overall structure of southern Marmara is composed of northeast trending right lateral strikeslip segments and northwest trending normal faults. They are components of large pull-apart structures in which Lakes Manyas and Ulubat, and the plains of Bursa, Yenişehir, and Gölpazarı are located (Figures 1 and 2a). The pull-apart structures decrease in size towards the east, probably due to the diminishing effect of extensional tectonics in western Turkey. Interestingly, this mimics the overall structure of NAFZ recognized by Şengör et al. (2005).

The Manyas-Karacabey Fault limits the eastern border of the Manyas pull-apart, showing an $8.70 \mathrm{~km}$ right lateral displacement of the Koca stream north of Manyas (Figure 2c). Further to the $\mathrm{NE}$, the Çapaz creek is diverted $5.70 \mathrm{~km}$ rightlaterally, immediately to the south of Karacabey (Figure 2d). The Manyas-Karacabey Fault has not been recognized in previous studies (e.g. Selim and Tüysüz, 2013). The eastern border of the Ulubat pull-apart basin is limited by the Ulubat Fault (Emre et al., 1997), which corresponds a 2.6 $\mathrm{km}$ right lateral diversion of the Nilüfer stream at southwest of Bursa (Figure 2e). After the northwest - southeast trending Bursa normal fault (Emre et al., 1997), the southern branch continues northeast with the Yıldirım Fault having $1.22 \mathrm{~km}$ right lateral displacement on Kaplikaya creek between Bursa and Kestel (Figure 2f). The southern branch then continues northeast through the western strike-slip boundary of the Yenişehir pull-apart basin (Y1lmaz and Koral, 2007; Gürbüz, 2010) (Figure 2a). The strike-slip faults on the eastern border of this basin have a northeasterly trend. This direction is somewhat compatible with the suggestion of Barka and Kadinsky-Cade (1988) that the southern branch joins the middle branch via Mekece (Figures 1 and 2a, black dotted lines). However, our findings indicate that the strike-slip faults on the eastern border of the Yenişehir pullapart basin bend from northeast to east northeast and are composed of several en-echelon segments that have right lateral diversions of the Göksu $(3.25 \mathrm{~km})$ and Çoklum creeks $(0.48 \mathrm{~km})$ (Figure $2 \mathrm{~g}$ ). Another example is the $2.93 \mathrm{~km}$ offset of the Sakarya River on Bayırköy Fault (Figure 2h). We are not certain, however, whether the deflections on the rivers/streams have been created by the fault segments or whether the rivers/streams follow the route of existing faults. The southern branch then moves southeast again, creating the western border of the Gölpazarı pull-apart basin (Figure 
2a) which is an important structure indicating the location of the main strike-slip branch (see earlier). The southern branch builds a releasing bend towards the east, passes through Mudurnu, and connects to the main branch of the NAFZ just south of the Bolu plain (Figures $1 \mathrm{~b}$ and $2 \mathrm{a}$ ). Evidence for the existence of the Mudurnu Fault is clearly seen in the MT section of Kaya (2010, p. 238) and we suggest it connects to the main branch of the NAFZ in a flower structure (Figure 1c).

\section{SEISMICITY}

Historical records indicate this new route for the southern branch has an active seismic history except at its most eastern end (Figure 1b). According to data provided by Tan et al. (2008), several remarkable historical earthquakes occurred along the southern branch (see Table 1), some of which were strong and destructive. Among these, the $\mathrm{AD} 160$ earthquake $(\mathrm{Ms}=7.1)$ occurred between Yenice and Gönen, and the February 28, 1855 earthquake $(\mathrm{Ms}=7.1)$ in Bursa caused extensive damage (Ambraseys, 2002; 2009).

Although historical earthquakes have large location errors, they are in agreement with recent instrumental seismicity (Figures $1 \mathrm{~b}$ and 2a). Several more recent major earthquakes $(M \geq$ 6.0) have also occurred along this branch of the NAFZ. The largest two events are the March 18, 1953 Yenice-Gönen earthquake $(\mathrm{Ms}=7.1)$ and the October 6, 1964 Manyas earthquake $(\mathrm{Ms}=6.8)$ (Ambraseys, 2002). Several moderate earthquakes $(5 \leq \mathrm{M}<6)$ have also been documented in the region (Figure 1b). In order to refine the location of the southern branch, recent earthquakes with a magnitude lower than 5.0 have been relocated and analysed for fault plane solutions (Figure $1 \mathrm{~b}$ and see Table 1).
We calculated relocation and fault plane solutions for several large earthquakes between 1982 and 2003 using bulletin data obtained from the International Seismological Centre (ISC) and for numerous small-scale earthquakes from 2004 to present using digital waveform data provided by Kandilli Observatory and the Earthquake Research Institute (KOERI).

After relocation, most events are compatible with the new southern branch (Figure 1b). Only a few earthquakes with large location errors are outside of the fault tracks due to lack of observation data or phase reading errors. All earthquakes were relocated with an average number of observations of $\sim 49$. The mean station gap in azimuth is $\sim 73^{\circ}$ and calculated errors in horizontal and vertical directions are 0.6 and 0.3 $\mathrm{km}$ respectively. The average RMS (root mean square) residual is $0.24 \mathrm{~s}$. All minimized location and origin time errors show that the seismicity of the region correlates with the active fault system.

Correlation between seismicity and local tectonics was investigated by looking at the fault plane solutions of selected earthquakes that occurred on the southern branch. We determined the individual focal mechanisms by using $\mathrm{P}$-wave first motion polarities. In the Table, focal mechanisms with a high quality solution are listed, they all tend to have relatively high station distribution values (STDR column) ( $\sim 0.57$ in average). Average misfit for all solutions is 0.06 . These results indicate that the focal mechanism solutions are reliable. The focal mechanism solutions on the new route for the southern branch show dominantly northwest southeast compression and northeast - southwest extension directions. Other than a small minority of solutions, these dominant regional stress directions are compatible with the pull-apart nature of the right lateral strike slip motion for the new southern branch (Figure 1b). 
Gürol SEYITOĞLU, Bülent KAYPAK, Bahadır AKTUĞ, Esra GÜRBÜZ, Korhan ESAT, Alper GÜRBÜZ

\begin{tabular}{|c|c|c|c|c|c|c|c|c|c|c|c|c|c|c|c|c|c|c|c|c|c|c|c|c|}
\hline 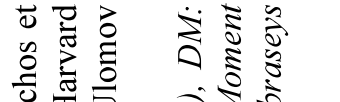 & & $\overleftrightarrow{\Xi}$ & & $\vec{y}$ & $\overleftrightarrow{z}$ & $\vec{y}$ & $\mathbb{z}$ & $\overleftrightarrow{z}$ & $\overrightarrow{2}$ & $\overleftrightarrow{z}$ & $\overleftrightarrow{z}$ & $\overleftrightarrow{z}$ & $\overleftrightarrow{z}$ & 岃 & $\overleftrightarrow{z}$ & そ & 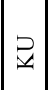 & ঊ & ₹ & & 2 & 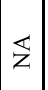 & $\overleftrightarrow{z}$ & $\overleftrightarrow{z}$ \\
\hline$\ddot{z} \bar{\sigma} \cong \bar{a}$ & & y৫LS & & , & . & . & 1 & ' & . & . & 1 & & & & & & & & & & & & & \\
\hline 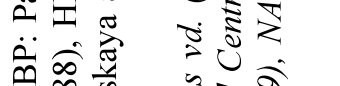 & & Iys!W & & . & . & . & 1 & $\cdot$ & 1 & ' & . & 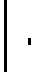 & & & . & & & & & & ' & & ' & \\
\hline$\stackrel{0}{\infty} \quad \frac{0}{0}=0$ & $z_{0}^{n}$ & $\mathrm{u}_{\mathrm{B} \#}$ & & . & . & . & I & . & 1 & . & . & & & I & . & & ' & & & & I & & & \\
\hline 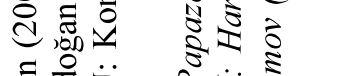 & \begin{tabular}{|l}
5 \\
3 \\
0 \\
0
\end{tabular} & $\begin{array}{c}\text { (०) } \\
\text { x৮yd }\end{array}$ & & ' & ' & . & I & . & & ' & , & & & & & & & & & & & & & \\
\hline 空证 & $\sum_{2}$ & (०) dIC & & . & . & . & . & . & ' & ' & . & I & & . & . & 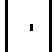 & ' & & ' & & , & . & 1 & \\
\hline 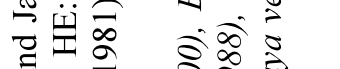 & $\begin{array}{l}\Xi \\
\text { 吾 } \\
\end{array}$ & $\begin{array}{c}\text { (०) } \\
\text { XLSA }\end{array}$ & & . & ' & . & ' & $\cdot$ & 1 & ' & ' & & & ' & ' & & & & & & 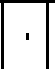 & & & \\
\hline 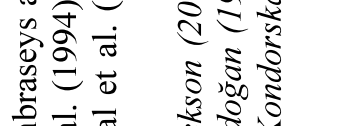 & 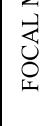 & 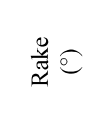 & & . & . & . & I & . & ' & ' & ' & & & & & & & & & & & & & \\
\hline 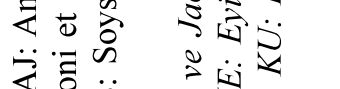 & & 言す & $\pi$ & ' & . & . & ' & $\cdot$ & ' & ' & ' & & & & & & & & & & & & & \\
\hline 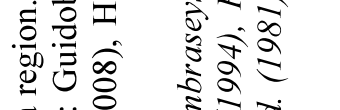 & & 窇气 & 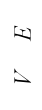 & . & 1 & I & 1 & . & 1 & 1 & 1 & ' & & ' & ' & & ' & & 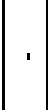 & & 1 & & & \\
\hline 돈 & & 画舜 & 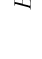 & . & . & . & . & . & . & ' & . & , & ' & . & . & . & . & ' & ' & & . & ' & ' & ' \\
\hline$a_{0}^{\infty}=$ & & 画衰 & $\omega$ & . &. & ' & ' & . & ' & ' & . & ' & ' & ' & ' & ' & . & ' & ' & & . & ' & ' & ' \\
\hline च & & $\sum_{a}^{n} \widehat{n}$ & $\pi$ & . & . & . & . & . & ' & , & . & ' & ' & ' & . & ' & ' & I & . & , & ' & ' & I & . \\
\hline$\sum_{-1}^{5}$ & & 突代 & - & . & . & . & . & . & ' & ' & ' & & & 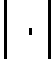 & 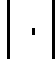 & & & & & & & & & \\
\hline 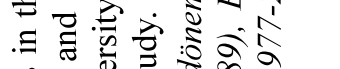 & & 㖼 & $\approx$ & . & ' & . & . & . & . & . & . & ' & ' & ' & . & ' & . & ' & ' & ' & . & . & , & . \\
\hline 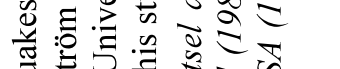 & $n$ & $\sum_{\Sigma}^{\infty}$ & 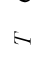 & 常 & \begin{tabular}{|l|} 
\\
\end{tabular} & 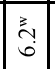 & 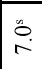 & $\frac{1}{n}$ & 离 & $\stackrel{m}{n}$ & \begin{tabular}{|l|} 
\\
$\infty$ \\
0 \\
0
\end{tabular} & $\begin{array}{ll} \\
0 \\
0 \\
0\end{array}$ & 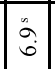 & $\begin{array}{ll} \\
\end{array}$ & $\begin{array}{ll}0 \\
0 \\
i\end{array}$ & 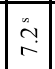 & \begin{tabular}{|l|} 
\\
\\
\\
\end{tabular} & $\begin{array}{ll} \\
\dddot{\sim} \\
\end{array}$ & 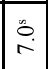 & 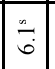 & \begin{tabular}{|l|}
$\vdots$ \\
\\
0
\end{tabular} & $\frac{m}{\sim}$ & 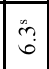 & $\begin{array}{l}n \\
i \\
i n\end{array}$ \\
\hline 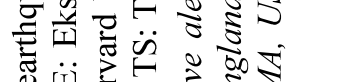 & 员 & 营言 & $n$ & . & ' & . & I & . & & ' & ' & & & & & & & & & & $\cong$ & & & \\
\hline 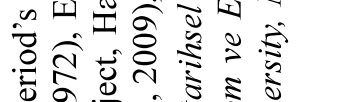 & 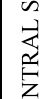 & ‡ే & 2 & $\begin{array}{l}8 \\
\text { ñ } \\
\text { iे }\end{array}$ & $\mid \begin{array}{l}8 \\
0 \\
n \\
0 \\
m \\
m\end{array}$ & $\mid \begin{array}{c}\stackrel{8}{0} \\
\infty \\
i \\
i\end{array}$ & 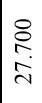 & 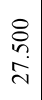 & $\begin{array}{l}8 \\
\infty \\
\infty \\
i\end{array}$ & 总 & $\mid \begin{array}{l}8 \\
0 \\
n \\
\dot{2} \\
m\end{array}$ & $\begin{array}{l}0 \\
\infty \\
\stackrel{\sim}{\sim}\end{array}$ & $\mid \begin{array}{c}8 \\
0 \\
\grave{\sim}\end{array}$ & $\left|\begin{array}{c}\infty \\
\infty \\
\stackrel{\sim}{\sim}\end{array}\right|$ & $\left|\begin{array}{l}8 \\
i n \\
0 \\
0 \\
n\end{array}\right|$ & $\mid \begin{array}{c}8 \\
\infty \\
\stackrel{\sim}{\sim}\end{array}$ & 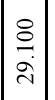 & \begin{tabular}{|l|} 
\\
$\infty$ \\
$\stackrel{\sim}{\sim}$
\end{tabular} & $\mid \begin{array}{l}8 \\
e \\
i \\
\end{array}$ & $\mid \begin{array}{l}8 \\
0 \\
0 \\
i \\
i\end{array}$ & $\mid \begin{array}{l}8 \\
\vdots \\
\infty \\
i \\
\end{array}$ & $\begin{array}{l}8 \\
0 \\
0 \\
i \\
i\end{array}$ & $\begin{array}{c}8 \\
\vdots \\
\infty \\
i \\
\end{array}$ & 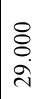 \\
\hline$\underbrace{8}_{n} \frac{0}{5}$ & 足 & 壱す & & 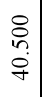 & $\mid \begin{array}{c}8 \\
0 \\
\vdots \\
\dot{y}\end{array}$ & 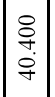 & 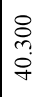 & $\begin{array}{l}\vdots \\
\vdots \\
\dot{+}\end{array}$ & $\begin{array}{l}8 \\
\substack{q \\
g}\end{array}$ & $\begin{array}{l}: \\
: \\
\dot{q}\end{array}$ & $\mid \begin{array}{c}8 \\
0 \\
0 \\
\vdots \\
q\end{array}$ & $\frac{8}{9}$ & 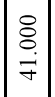 & $\mid \begin{array}{l}a \\
\dot{q} \\
\dot{q} \\
\end{array}$ & $\mid \begin{array}{c}0 \\
0 \\
0 \\
\vdots \\
q\end{array}$ & $\mid \begin{array}{c}8 \\
⿱ 亠 䒑 \\
⿱ 亠 巾 \\
q\end{array}$ & $\mid \begin{array}{c}0 \\
\stackrel{0}{a} \\
\dot{q}\end{array}$ & $\begin{array}{c}8 \\
⿱ 亠 䒑 \\
\vdots \\
\dot{q}\end{array}$ & $\frac{8}{9}$ & $\mid \begin{array}{c}8 \\
\stackrel{9}{9} \\
\end{array}$ & $\begin{array}{l}8 \\
0 \\
\dot{q} \\
\end{array}$ & $\frac{8}{\stackrel{9}{9}}$ & 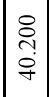 & $\begin{array}{l}8 \\
\dot{0} \\
\dot{q}\end{array}$ \\
\hline 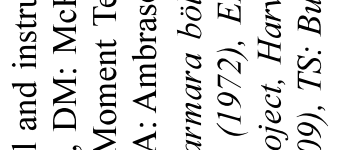 & & 惫兽 & & ' & I & 1 & 1 & . & (. & ' & . & ' & ' & & $\mid \begin{array}{c}0 \\
\dot{0} \\
\dot{0} \\
\dot{j} \\
\dot{d}\end{array}$ & $\mid \begin{array}{c}0 \\
\stackrel{0}{0} \\
\dot{0} \\
\dot{i} \\
\dot{\sim}\end{array}$ & & $\left|\begin{array}{c}0 \\
\dot{0} \\
0 \\
0 \\
\vdots \\
\vdots\end{array}\right|$ & & 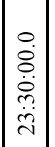 & & & $\left|\begin{array}{c}0 \\
\dot{0} \\
\dot{q} \\
\dot{q} \\
\dot{2}\end{array}\right|$ & $\begin{array}{l}0 \\
\dot{0} \\
\dot{0} \\
\dot{0}\end{array}$ \\
\hline 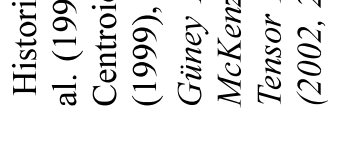 & & 逽 & & $\begin{array}{l}0 \\
0 \\
0 \\
0 \\
0 \\
0\end{array}$ & 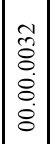 & 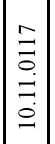 & 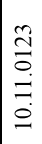 & $\begin{array}{l}0 \\
0 \\
0 \\
0 \\
0 \\
0\end{array}$ & $\begin{array}{ll}8 \\
0 \\
0 \\
0 \\
0 \\
0 \\
0\end{array}$ & 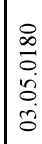 & $\mid \begin{array}{l}\infty \\
0 \\
0 \\
0 \\
0 \\
= \\
=\end{array}$ & $\mid \begin{array}{l}\infty \\
0 \\
0 \\
\\
\\
\\
8\end{array}$ & $\left|\begin{array}{l}0 \\
0 \\
0 \\
0 \\
0 \\
0 \\
8\end{array}\right|$ & 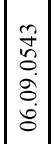 & 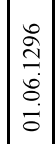 & 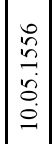 & $\mid \begin{array}{l}1 \\
5 \\
0 \\
0 \\
0 \\
8 \\
\end{array}$ & $\mid \begin{array}{l}0 \\
0 \\
0 \\
0 \\
0 \\
0\end{array}$ & 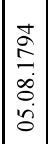 & $\mid \begin{array}{c}0 \\
0 \\
\infty \\
\dot{0} \\
\dot{0} \\
\dot{2}\end{array}$ & $\mid$\begin{tabular}{|c|}
$\overrightarrow{0}$ \\
$\infty$ \\
$\dot{8}$ \\
$\dot{8}$ \\
\end{tabular} & 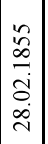 & 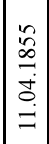 & 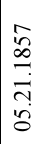 \\
\hline 总 & & $z_{z}$ & & $\vec{I}$ & $\approx$ & 9 & 造 & 告 & : & I & $\stackrel{\infty}{\Psi}$ & 社 & 岦 & $\bar{\nabla}$ & $\cong$ & $\stackrel{m}{ \pm}$ & $\vec{\Psi}$ & $\stackrel{n}{\Xi}$ & $\stackrel{0}{ \pm}$ & 全 & $\stackrel{\infty}{ \pm}$ & 言 & ปิ) & $\overrightarrow{\underline{x}}$ \\
\hline
\end{tabular}




\begin{tabular}{|c|c|c|c|c|c|c|c|c|c|c|c|c|c|c|c|c|c|c|c|c|c|c|c|c|c|c|c|c|c|c|c|c|c|c|}
\hline$\underset{z}{\sharp}$ & $\overleftrightarrow{z}$ & $\mathbb{z}$ & $\mathbb{z}$ & 里 & $\overleftrightarrow{z}$ & z & $\mid \mathbb{z}$ & & & & 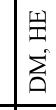 & $\begin{array}{l}\text { 䛼 } \\
\sum \\
\text { 品 }\end{array}$ & $\sum$ & \begin{tabular}{|l|} 
毀 \\
品
\end{tabular} & 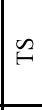 & $\omega$ & $\hat{m}$ & 贸 & $\vec{y}$ & 栾 & $\stackrel{\varrho}{\oplus}$ & 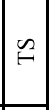 & 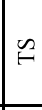 & 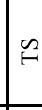 & $\tilde{\omega}$ & 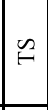 & $\stackrel{\infty}{\epsilon}$ & 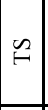 & $\tilde{\omega}$ & 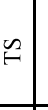 & $\stackrel{\Omega}{\bullet}$ & 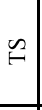 & 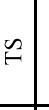 & 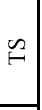 \\
\hline & ' & & & & & & & & ' & ' & ' & ' & , & & $\vec{m}$ & f. & & & & & $\begin{array}{c}\hat{n} \\
0 \\
\end{array}$ & $\begin{array}{l}0 \\
: \\
0 \\
\end{array}$ & 定 & $\stackrel{?}{?}$ & $\begin{array}{l}0 \\
: \\
0 \\
0\end{array}$ & $\begin{array}{l}9 \\
0 \\
0\end{array}$ & \begin{tabular}{|c|}
$\infty$ \\
$\tilde{0}$ \\
0
\end{tabular} & $\begin{array}{l}\overrightarrow{0} \\
0 \\
\end{array}$ & $\begin{array}{l}n \\
n \\
0\end{array}$ & $\begin{array}{l}\vec{b} \\
0 \\
0\end{array}$ & $\begin{array}{l}0 \\
\text { n. } \\
0\end{array}$ & $\begin{array}{l}7 \\
5 \\
0\end{array}$ & 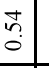 & $\widehat{\widehat{A}}$ \\
\hline & ' & & & & & & & & & ' & & ' & ' & & ${ }_{0}^{0}$ & 8 & & & & & $\mid \begin{array}{l}0 \\
0 \\
0\end{array}$ & $\begin{array}{l}8 \\
0 \\
0\end{array}$ & $\stackrel{8}{\circ}$ & $\stackrel{8}{\circ}$ & $\mid \begin{array}{l}\infty \\
0 \\
0\end{array}$ & $\begin{array}{l}0 \\
0 \\
0\end{array}$ & $\begin{array}{l}= \\
0\end{array}$ & $\div$ & $\simeq$ & 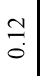 & $\begin{array}{l}8 \\
0 \\
0\end{array}$ & $\begin{array}{l}8 \\
: \\
0\end{array}$ & 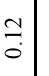 & $\stackrel{8}{\circ}$ \\
\hline & '] & & & & & & & & ' & ' & ' & ' & ' & & \pm & 6 & & & & & $f$ & $\div$ & $\circ$ & $=$ & $\approx$ & $=$ & \pm & $\stackrel{\infty}{\sim}$ & \pm & $\dot{\sim}$ & $=1$ & $\simeq$ & $q$ & \\
\hline & $'$ & & & & & & & & ' & ' & ' & ' & ' & & 0 & 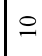 & & & & ' & 0 & $\circ$ & ¿ & 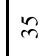 & i & in & \& & $\stackrel{\sim}{\sim}$ & i & $\simeq$ & $\therefore$ & 이 & i & \\
\hline & ' & & & & & & & & ' & ' & , & ' & ' & & $\cong$ & in & & & & ' & $\infty$ & $\circ$ & $\approx$ & in & $m$ & $\circ$ & $\cong$ & $=$ & in & $\circ$ & $m$ & in & in & $\therefore$ \\
\hline & . & & & & & & & & ' & ' & , & ' & ' & & in & in & & & & ' & in & f & in & $m$ & in & 0 & 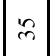 & $\infty$ & in & $\infty$ & in & $\simeq$ & $n$ & $\infty$ \\
\hline & 1 & & & & & & & $\sim$ & & ' & 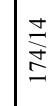 & 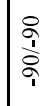 & 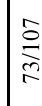 & $\frac{\text { g }}{\frac{8}{d}}$ & 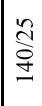 & $\begin{array}{l}\infty \\
\stackrel{\infty}{\infty} \\
\stackrel{2}{0}\end{array}$ & 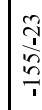 & $\begin{array}{l}\stackrel{0}{0} \\
\frac{\infty}{1} \\
\end{array}$ & $\mid \overline{\frac{7}{1}}$ & $\begin{array}{l}\overrightarrow{\widetilde{T}} \\
\frac{1}{0} \\
\stackrel{7}{1}\end{array}$ & 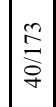 & 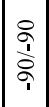 & 管 & 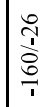 & 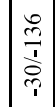 & 产 & $\frac{\mathcal{z}}{\frac{1}{2}}$ & $\begin{array}{c}\frac{2}{2} \\
\frac{1}{2} \\
\frac{n}{1}\end{array}$ & $\mid \begin{array}{c}m \\
\grave{D} \\
\bar{n}\end{array}$ & $\begin{array}{l}\frac{9}{9} \\
\stackrel{0}{7}\end{array}$ & 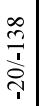 & $\begin{array}{l}\vdots \\
\vdots \\
0\end{array}$ & $\begin{array}{l}\frac{m}{b} \\
\end{array}$ & $\begin{array}{l}0 \\
\frac{1}{1} \\
\frac{1}{2}\end{array}$ \\
\hline & ' & & & & & & & $z$ & ' & ' & 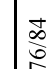 & 芯 & 守 & $\begin{array}{l}n \\
0 \\
0\end{array}$ & $\frac{\Re}{2}$ & \begin{tabular}{|l}
$\bar{n}$ \\
$\bar{g}$
\end{tabular} & $\stackrel{\circ}{\circ}$ & $\frac{2}{2}$ & 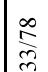 & \begin{tabular}{l}
$\infty$ \\
\multirow{2}{*}{}
\end{tabular} & 号 & 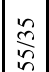 & \begin{tabular}{|l}
$\vec{\infty}$ \\
$\hat{s}$
\end{tabular} & 於 & $\begin{array}{l}5 \\
0 \\
0\end{array}$ & 妾 & $\begin{array}{l}\stackrel{2}{2} \\
\Sigma\end{array}$ & $\frac{m}{5}$ & 离 & 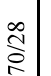 & 吕 & $\frac{\pi}{\frac{2}{3}}$ & $\bar{n}$ & 第 \\
\hline & & & & & & & & $\vec{\Delta}$ & & & $\frac{8}{2}$ & 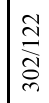 & ฐ্ & 芯 & $\stackrel{\circ}{\stackrel{2}{2}}$ & 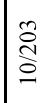 & 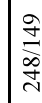 & 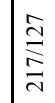 & $\begin{array}{l}\frac{\tilde{F}}{\mathrm{i}} \\
\text { ले }\end{array}$ & $\begin{array}{l}\stackrel{0}{0} \\
\stackrel{0}{0} \\
\stackrel{0}{0}\end{array}$ & 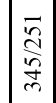 & 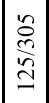 & $\begin{array}{l} \\
\text { 离 } \\
\text { on } \\
\text { on }\end{array}$ & 을 & 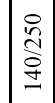 & 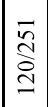 & $\frac{n}{\frac{n}{s}}$ & $\begin{array}{l}0 \\
\frac{c}{2} \\
\frac{v}{0}\end{array}$ & $\frac{\vec{m}}{2}$ & 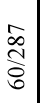 & 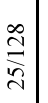 & ָิ & 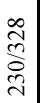 & $\stackrel{\infty}{\infty}$ \\
\hline & ' & & & & & & & & ' & . & . & . & . & & in & 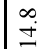 & & , & & , & $\mid \begin{array}{l}0 \\
=\end{array}$ & $\ddot{0}$ & $\because$ & $\because$ & $\because$ & $\overrightarrow{0}$ & $\overrightarrow{0}$ & $\because$ & $\because$ & $\overrightarrow{0}$ & $\overrightarrow{0}$ & 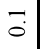 & $\overrightarrow{0}$ & $\stackrel{3}{0}$ \\
\hline & ' & & & & & & & $\nabla$ & ' & ' & ' & ' & ' & & $\stackrel{m}{m}$ & @ֶ: & & 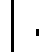 & 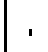 & ' & $\stackrel{1}{\beth}$ & $\begin{array}{l}n \\
0\end{array}$ & Fo & F & $\tilde{o}$ & $\begin{array}{l}m \\
0\end{array}$ & $\begin{array}{l}1 \\
0\end{array}$ & $\begin{array}{l}1 \\
0\end{array}$ & \begin{tabular}{|l|} 
\\
0
\end{tabular} & 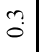 & $\begin{array}{l}0 \\
\circ\end{array}$ & $?$ & $\begin{array}{ll} \\
0\end{array}$ & $\stackrel{0}{\circ}$ \\
\hline & . & & & । & & & & i & ' & ' & 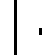 & ' & ' & & $\underset{m}{\infty}$ & 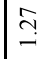 & & ' & & ' & $\overrightarrow{\tilde{m}}$ & $\begin{array}{l}7 \\
0\end{array}$ & $\stackrel{\circ}{\circ}$ & $\underset{\Delta}{0}$ & $\stackrel{0}{0}$ & $\frac{7}{0}$ & $\overline{0}$ & $\stackrel{0}{0}$ & $\left|\begin{array}{c}\infty \\
0 \\
0\end{array}\right|$ & $\stackrel{?}{\circ}$ & $\stackrel{\infty}{0}$ & $\begin{array}{c}m \\
0 \\
0\end{array}$ & ¿ุ| & $\overrightarrow{0}$ \\
\hline & ' & & & I & & & & $\Leftrightarrow$ & . & , & . & . & 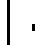 & & సิ & f & & ' & ' & ' & $=$ & $\approx$ & to & in & $\nsubseteq$ & $\widehat{\cong}$ & \pm & $\infty$ & $\approx$ & లు & $\Xi$ & $\infty$ & $n$ & $\exists$ \\
\hline & ' & & & & & & & $z$ & . & . & . & . & 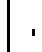 & & $\stackrel{\infty}{\rightarrow}$ & $m$ & & ' & . & ' & $\vec{m}$ & तิ & 2 & $\stackrel{2}{2}$ & $m$ & $\approx$ & $\vec{m}$ & $m$ & ते & $\approx$ & $\vec{\sim}$ & $\stackrel{\sim}{\sim}$ & $\ddot{0}$ & 2 \\
\hline in & 总 & $\begin{array}{l}0 \\
\text { in }\end{array}$ & $\begin{array}{l}\text { o. } \\
\text { in }\end{array}$ & in & $\left|\begin{array}{l}0 \\
0 \\
0 \\
0\end{array}\right|$ & 总 & \begin{tabular}{|c}
0 \\
$i$ \\
$i n$
\end{tabular} & $\approx$ & 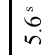 & $\begin{array}{l}n \\
\vec{b} \\
i n\end{array}$ & $\stackrel{\text { }}{\stackrel{\circ}{r}}$ & 离 & 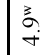 & $\begin{array}{l}\underbrace{z}_{0} \\
\text { in }\end{array}$ & $\underset{m}{\stackrel{m}{q}}$ & $\begin{array}{l}\stackrel{\infty}{\infty} \\
+ \\
+\end{array}$ & $\frac{n}{6}$ & 妾 & ث̈ & $\begin{array}{l}\overrightarrow{0} \\
\text { in } \\
\text { in }\end{array}$ & $\overrightarrow{\ddot{n}}$ & $\begin{array}{l}\vec{b} \\
\dot{m}\end{array}$ & $\stackrel{\Rightarrow}{m}$ & 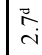 & $\underset{\tilde{N}}{\tilde{N}}$ & $\frac{\vec{m}}{m}$ & $\frac{\vec{m}}{m}$ & $\begin{array}{l}\vec{j} \\
\dot{m}\end{array}$ & $\stackrel{\vec{m}}{m}$ & $\vec{\sim}$ & $\stackrel{a}{\Delta}$ & $\stackrel{m}{m}$ & $\underset{\sim}{\stackrel{v}{*}}$ & 号 \\
\hline ' & ' & & & & & & & it & 8 & & $\stackrel{\circ}{\stackrel{0}{0}}$ & $\stackrel{\circ}{\odot}$ & $\stackrel{\circ}{\mathrm{I}}$ & $\stackrel{\circ}{+}$ & $\stackrel{i}{i}$ & - & 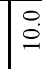 & in & $\stackrel{i}{i}$ & i & $\bar{\sigma}$ & $\begin{array}{l} \\
\text { in } \\
\end{array}$ & in & in & $\dot{\ddot{m}}$ & $\begin{array}{l}+ \\
\infty\end{array}$ & $\begin{array}{l} \\
\end{array}$ & 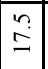 & 7 & $\stackrel{0}{\circ}$ & in & ir & $\therefore$ & $\vec{a}$ \\
\hline 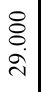 & 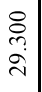 & 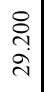 & 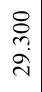 & $\begin{array}{l}\stackrel{8}{a} \\
\infty \\
\infty \\
\sim\end{array}$ & $\mid \begin{array}{l}\circ \\
\stackrel{0}{0} \\
0\end{array}$ & $\begin{array}{l}8 \\
\infty \\
\infty \\
i\end{array}$ & 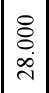 & $\begin{array}{l}z \\
-\end{array}$ & $\begin{array}{l}\text { \& } \\
\text { ते }\end{array}$ & 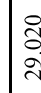 & 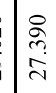 & 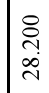 & 京 & 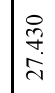 & 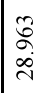 & 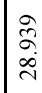 & 足 & $\begin{array}{l}\infty \\
\infty \\
\tilde{n} \\
\tilde{n}\end{array}$ & 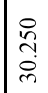 & 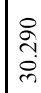 & $\mid \begin{array}{l}R \\
0 \\
\dot{d} \\
d\end{array}$ & $\mid \begin{array}{l}0 \\
\infty \\
\stackrel{\sim}{\sim}\end{array}$ & $\begin{array}{l}\vec{i} \\
\text { ते } \\
\text { in }\end{array}$ & 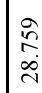 & $\mid \begin{array}{l}0 \\
\hat{\sigma} \\
\dot{v}\end{array}$ & $\mid \begin{array}{l}0 \\
\dot{0} \\
0 \\
\dot{m}\end{array}$ & 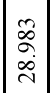 & 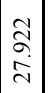 & 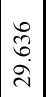 & 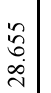 & $\begin{array}{l}0 \\
\infty \\
\stackrel{2}{\sim} \\
\stackrel{n}{n}\end{array}$ & 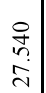 & $\begin{array}{l}\bar{\sigma} \\
\vec{v}\end{array}$ & 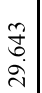 \\
\hline$\stackrel{8}{ }$ & $\begin{array}{l}\stackrel{8}{0} \\
\stackrel{+}{0}\end{array}$ & $\mid \begin{array}{l}0 \\
\dot{q} \\
\dot{q} \\
\end{array}$ & 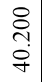 & $\begin{array}{l}8 \\
0 \\
\dot{q}\end{array}$ & $\mid \begin{array}{l}0 \\
0 \\
6 \\
\dot{q} \\
\end{array}$ & $\begin{array}{l}8 \\
\stackrel{\square}{\circ} \\
\dot{+}\end{array}$ & $\mid \begin{array}{l}8 \\
\dot{0} \\
\dot{q}\end{array}$ & & 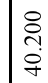 & 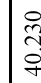 & $\mid \begin{array}{l}0 \\
0 \\
0 \\
\dot{y}\end{array}$ & 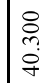 & ঙ্ল & $\mid \begin{array}{l}\mathcal{7} \\
\dot{q}\end{array}$ & \begin{tabular}{|l} 
in \\
wa \\
$\dot{q}$
\end{tabular} & $\begin{array}{l}\hat{0} \\
\dot{q}\end{array}$ & 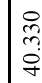 & $\begin{array}{l}0 \\
m \\
\\
\end{array}$ & $\begin{array}{l}\stackrel{8}{q} \\
\stackrel{\sigma}{\sigma}\end{array}$ & $\begin{array}{l}\frac{0}{m} \\
\stackrel{o}{q}\end{array}$ & $\mid \begin{array}{l}\mathbf{1} \\
0 \\
0 \\
0\end{array}$ & $\mid \begin{array}{c}0 \\
0 \\
0 \\
\dot{q}\end{array}$ & 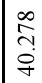 & $\begin{array}{l}\stackrel{8}{0} \\
\stackrel{0}{0} \\
\sigma\end{array}$ & $\mid \begin{array}{l}n \\
\infty \\
0 \\
\dot{\sigma}\end{array}$ & $\mid \begin{array}{c}0 \\
\tilde{g} \\
\dot{q}\end{array}$ & $\mid \begin{array}{c}\tilde{N} \\
\tilde{q} \\
\dot{q}\end{array}$ & $\left|\begin{array}{c}0 \\
0 \\
0 \\
\dot{q}\end{array}\right|$ & 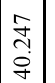 & $\begin{array}{l}\overrightarrow{0} \\
\dot{0} \\
\dot{q}\end{array}$ & $\begin{array}{l}n \\
\hat{\alpha} \\
\dot{q}\end{array}$ & $\begin{array}{l}\vec{b} \\
\dot{b} \\
\dot{q}\end{array}$ & $\begin{array}{l}\tilde{n} \\
\hat{\imath} \\
\dot{q}\end{array}$ & 8 \\
\hline & $\begin{array}{l}0 \\
\dot{0} \\
\dot{0} \\
\dot{0} \\
\dot{0}\end{array}$ & & 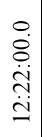 & & 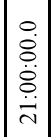 & $\begin{array}{l}\circ \\
\dot{0} \\
\dot{0} \\
\dot{=} \\
\dot{=}\end{array}$ & $\mid \begin{array}{l}0 \\
\dot{0} \\
\dot{0} \\
\dot{0} \\
0\end{array}$ & & 8 & & 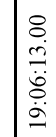 & 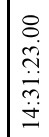 & $\begin{array}{l}\stackrel{8}{.} \\
\stackrel{+}{\dot{0}} \\
\stackrel{0}{0} \\
\end{array}$ & 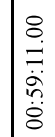 & 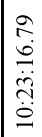 & 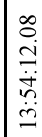 & 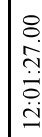 & $\begin{array}{l}- \\
\vec{g} \\
\dot{v} \\
\tilde{d} \\
\stackrel{i}{n}\end{array}$ & 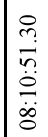 & $\begin{array}{l}\stackrel{8}{0} \\
\text { ñ. } \\
\hat{n} \\
=\end{array}$ & $\mid \begin{array}{l}a \\
0 \\
\dot{0} \\
\dot{+} \\
\dot{\Xi} \\
\vdots\end{array}$ & 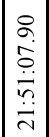 & 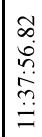 & 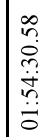 & $\mid \begin{array}{c}\infty \\
0 \\
\infty \\
\sim \\
\dot{0} \\
\dot{+} \\
\tilde{\sigma}\end{array}$ & 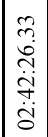 & 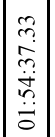 & $\mid \begin{array}{c}\bar{m} \\
\dot{0} \\
\grave{a} \\
\dot{o} \\
\dot{8}\end{array}$ & $\mid \begin{array}{l}n \\
\tilde{n} \\
\tilde{n} \\
\tilde{c} \\
\ddot{2}\end{array}$ & 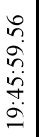 & $\begin{array}{l}\hat{N} \\
\dot{0} \\
\dot{y} \\
\dot{\vec{v}} \\
\dot{v}\end{array}$ & 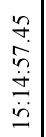 & $\begin{array}{c}\stackrel{\tilde{O}}{ } \\
\dot{\sim} \\
\ddot{n} \\
\ddot{\infty} \\
\stackrel{\infty}{0}\end{array}$ & t \\
\hline 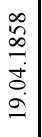 & $\begin{array}{l}0 \\
\stackrel{0}{0} \\
\dot{0} \\
\dot{0} \\
\dot{0}\end{array}$ & 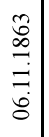 & 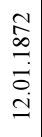 & 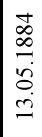 & 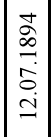 & 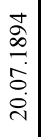 & 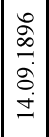 & & 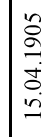 & $\begin{array}{l}\infty \\
\stackrel{d}{d} \\
= \\
=\end{array}$ & $\begin{array}{l}\tilde{n} \\
2 \\
\sigma \\
\infty \\
\infty \\
\infty\end{array}$ & 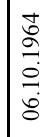 & 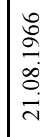 & 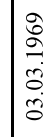 & 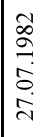 & 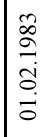 & 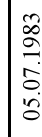 & 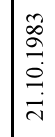 & 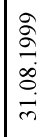 & 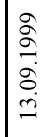 & 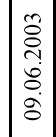 & 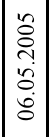 & 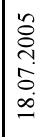 & 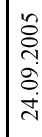 & 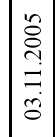 & 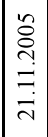 & 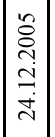 & 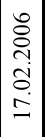 & 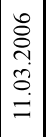 & 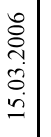 & $\begin{array}{l}0 \\
\stackrel{0}{0} \\
i ் \\
0 \\
\dot{0} \\
\dot{0}\end{array}$ & 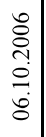 & & s. \\
\hline$\widetilde{\mathbb{Z}}$ & בี & Z & $\dddot{\Xi}$ & đ̊ & $\widehat{\hat{y}}$ & 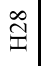 & ચి & & - & r & $m$ & $\nabla$ & in & 0 & - & $\infty$ & $a$ & 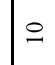 & $=$ & $\simeq$ & 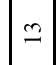 & \pm & 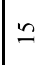 & ○ & $=$ & 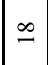 & 2 & $\stackrel{\sim}{ }$ & $\vec{\sim}$ & ป & $\vartheta$ & $\dot{4}$ & $\approx$ & 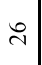 \\
\hline
\end{tabular}




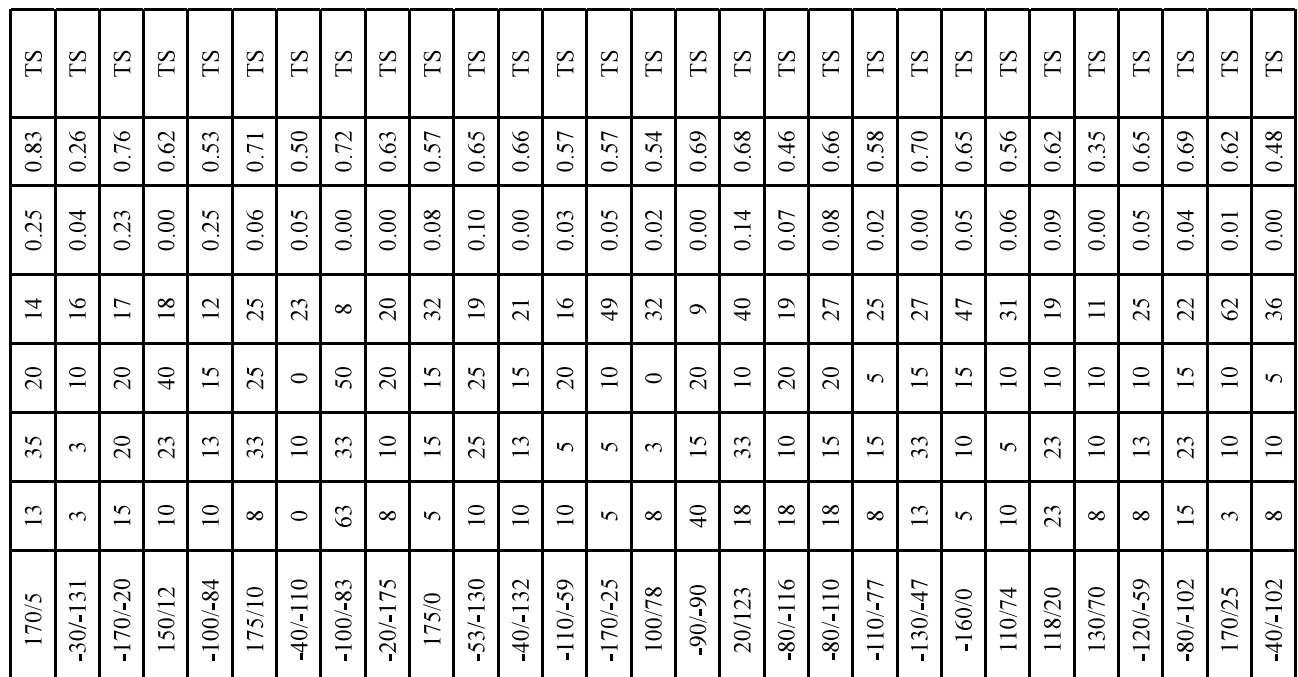

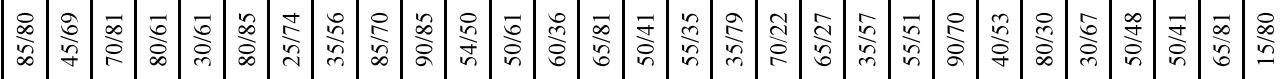

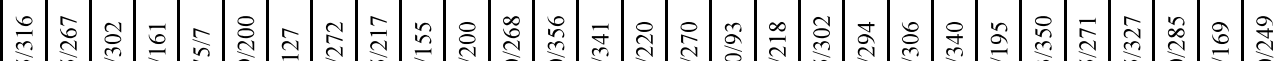

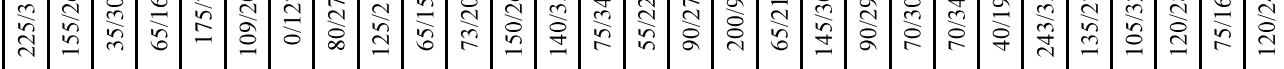

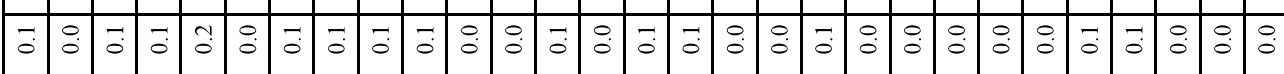

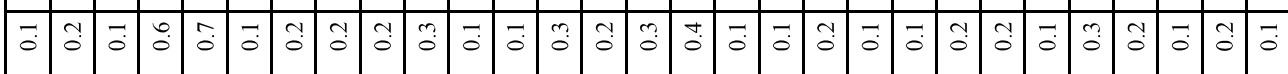

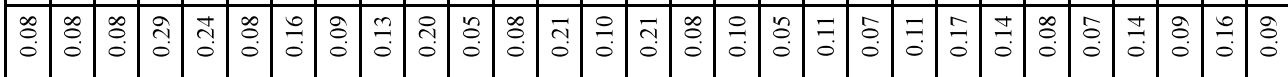

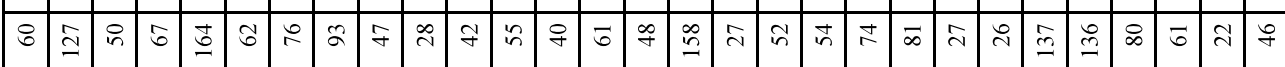

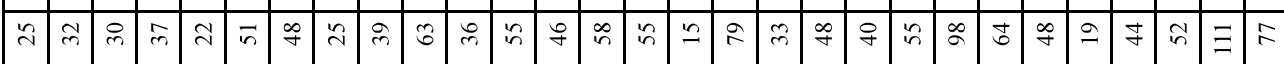

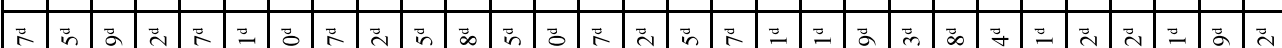

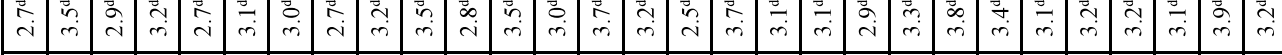

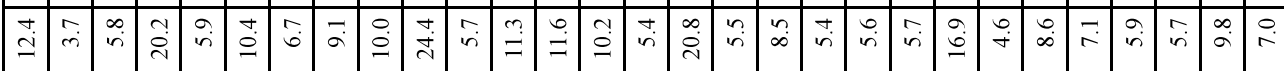
ว)

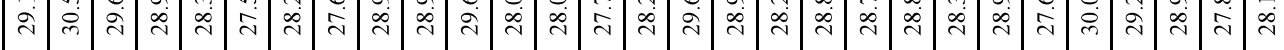

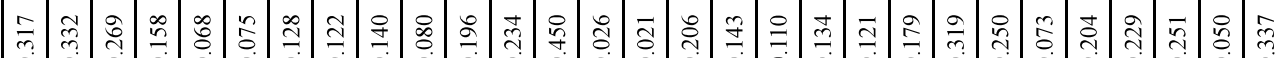

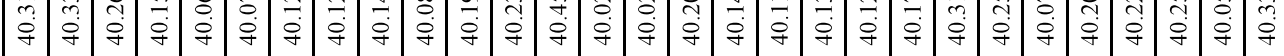

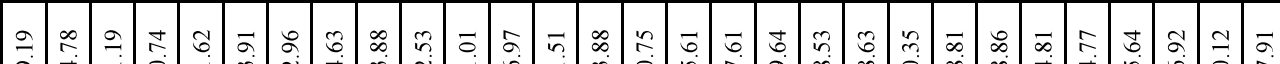
ᄀ่)

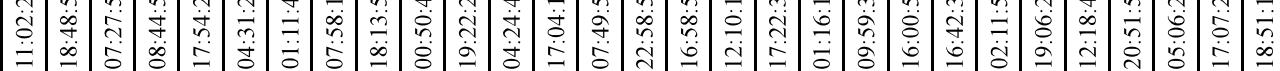

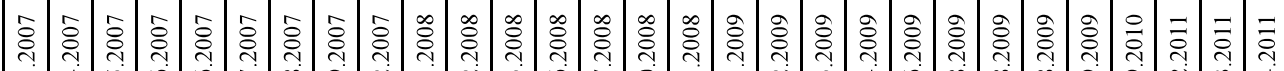

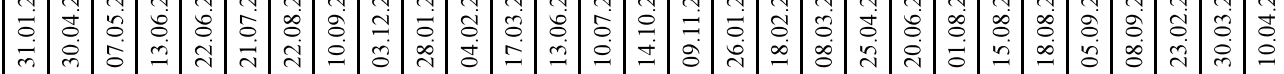

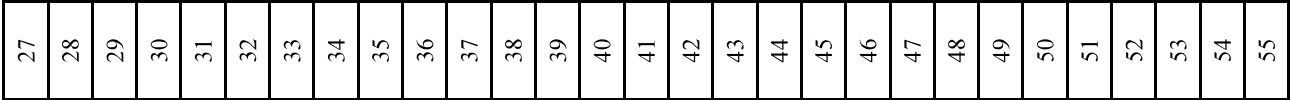




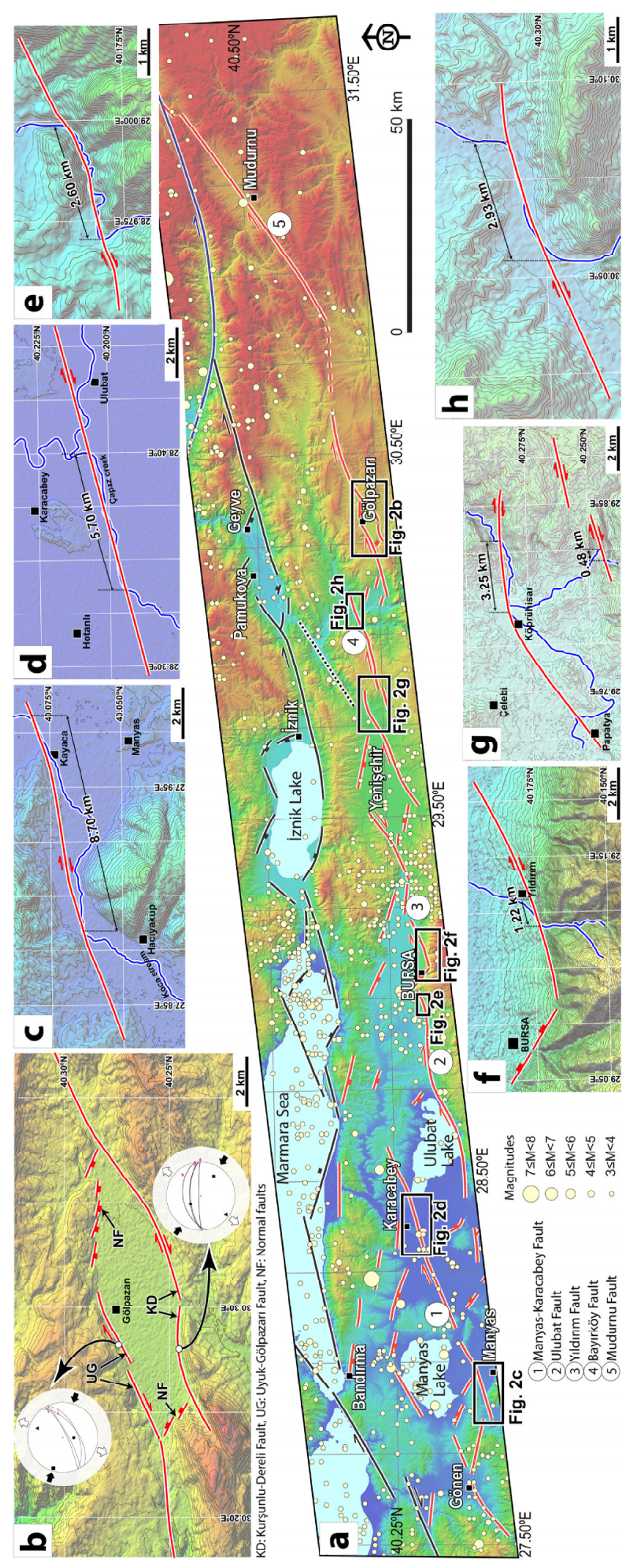

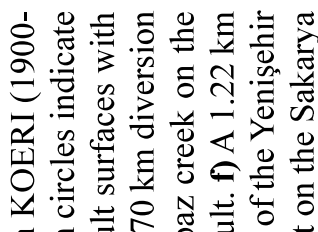

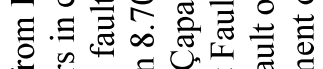

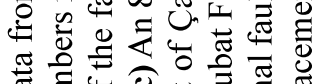

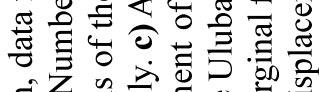

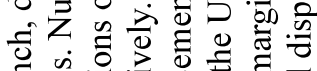

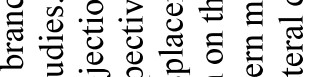
E 专

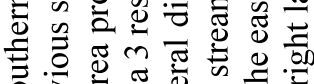

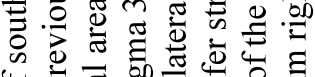

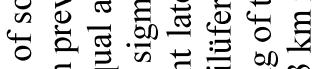

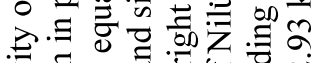

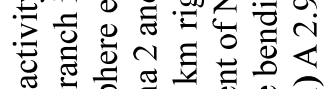

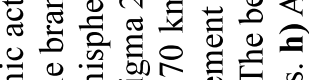

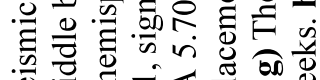

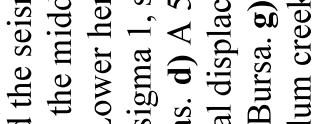

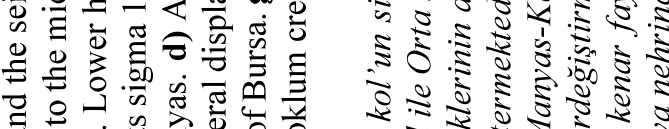

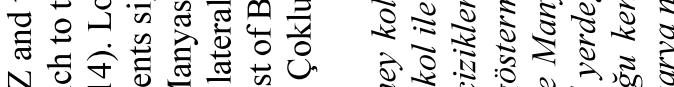

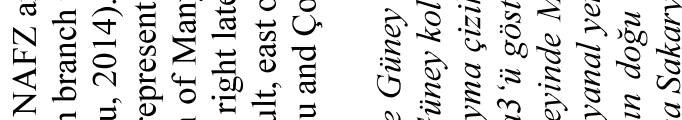

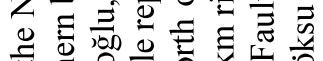

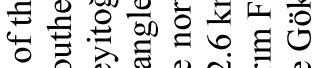

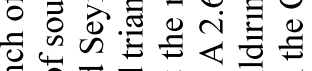

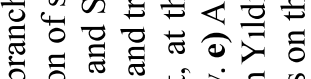

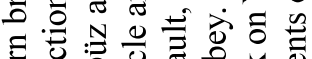

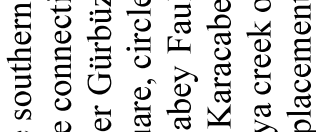

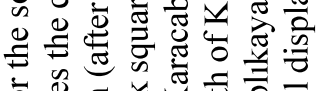

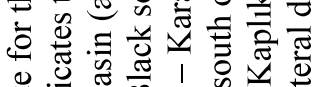

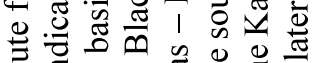

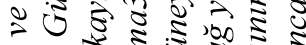

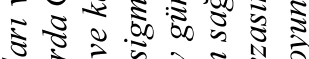

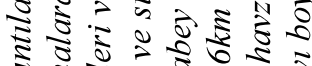

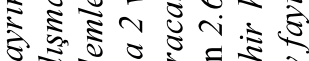

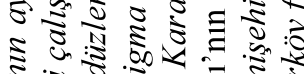

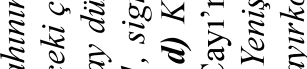
80.050

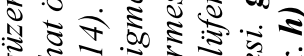

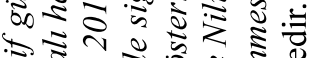

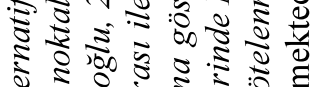

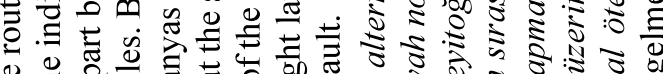

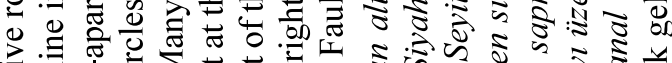

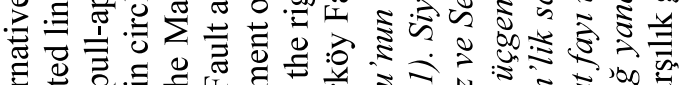

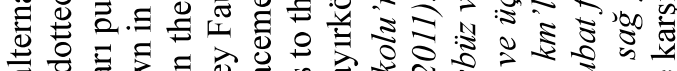

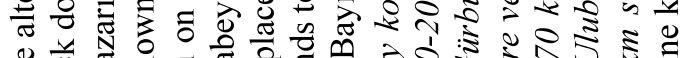

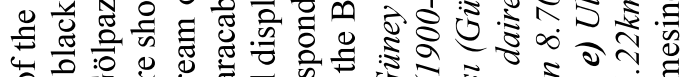
पू⿺辶寸

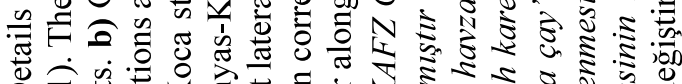

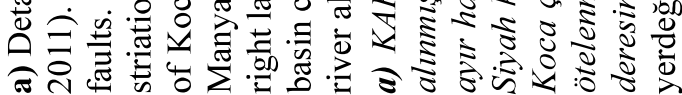

竞

$\frac{2}{5}$ 


\section{GPS DATA}

Slip partitioning in the Marmara region was previously examined by GPS data such that the computed slips rate range between 23 and $28 \mathrm{~mm} /$ $\mathrm{yr}$ and between 2.9 to $9.6 \mathrm{~mm} / \mathrm{yr}$ for the northern and southern branches, respectively (Meade et al., 2002; Nyst and Thatcher, 2004; Reilinger et al., 2006; Aktuğ et al., 2009; Le Pichon and Kreemer, 2010). The majority of these studies consider only two simplified branches of the NAFZ in the Marmara region. Flerit et al. (2004) found slip rates using three branches resulting in slip rates of 17-20, 5 and 2-5 mm/yr for the northern, middle, and southern branches respectively. We employed block modeling to compute the slip rates along boundaries of the fault mapping of this study and earlier works. In this approach, rigid-body motions of each block parameterized by Euler vectors are simultaneously estimated by minimizing the misfit between the modeled and the observed velocities (McCaffrey, 2002; 2005). To account for elastic straining along block boundaries (Okada, 1985), the back slip approach (Matsu'ura et al., 1986) was applied for each fault segment with prescribed fault geometry (Aktuğ et al., 2013b). We assumed vertical faults in Marmara with locking depths of $15 \mathrm{~km}$.

In order to examine slip distribution between the branches of the NAFZ, the Marmara region is divided into 5 blocks using previous fault definitions (Figure 3a). In this case, the northern branch of the NAFZ has the largest slip values (11.7-13.7 mm/yr) except for the northern part of the Almacik flake. The middle branch has the second largest slip rates, ranging from 2.1 to 5.1 $\mathrm{mm} / \mathrm{yr}$. In this configuration, the southern branch is the least important structure with low slip values, $(0.1-3.5 \mathrm{~mm} / \mathrm{yr})$ and the eastern segment of the southern branch has left-lateral slip rates that are inconsistent with the nature of the Yenişehir pull-apart basin and the entire right lateral nature of the NAFZ.

The newly proposed route of the southern branch in the Marmara region results in a better fit with the GPS velocities (Figure 3b). As shown in Figure 3b, the residuals in the southernmost block are much smaller than those in Figure 3a. The northern branch still has the highest slip rates $(11.2-15.8 \mathrm{~mm} / \mathrm{yr})$, but the new slip rates indicate that the new southern route has the next highest slip rates, ranging between 1.0 and 3.7 $\mathrm{mm} / \mathrm{yr}$. Block modeling with the newly proposed route provides a lower RMS value than using the geometry given in previous studies $(2.32 \mathrm{~mm} /$ yr versus $2.90 \mathrm{~mm} / \mathrm{yr}$ ). The results of the block modeling were further analyzed statistically to determine whether the improvement in RMS is significant. An F-ratio test of the variances of two different estimations reveals that such a difference in variances is statistically significant at a $95 \%$ confidence level. 

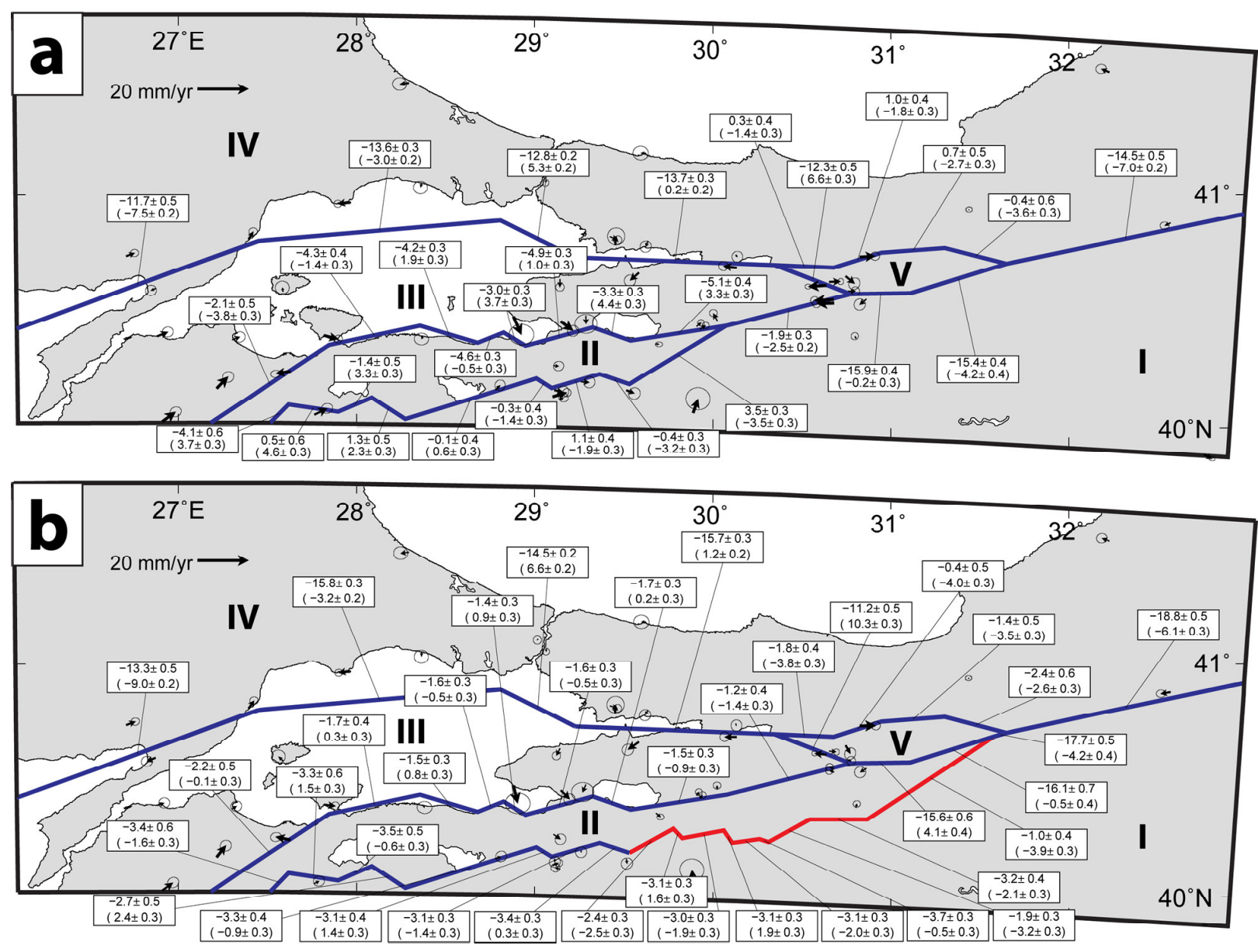

Figure 3. Slip distribution in the Marmara region. a) Block boundaries are determined by previous workers cited in Figure 1. b) Block boundaries are determined according to the new route of the southern branch of the NAFZ. The slip rates computed using the block model are shown in $\mathrm{mm} / \mathrm{yr}$. Top values without parentheses are strike-slip rates, positive being left-lateral. Numbers in parentheses are fault-normal slip rates, positive being closing. Error ellipses are at $95 \%$ confidence level.

Şekil 3. Marmara bölgesinde kayma dağıllımı. a) Blok sınırları Şekil 1'de referans gösterilen önceki çalışmalara göre tanımlanmıştır. b) Blok sınırları KAFZ'nun Güney Kol'unun yeni güzergahına göre tanımlanmıştır. Kayma değerleri blok modele göre hesaplanmış olup, $\mathrm{mm} / y$ ll olarak gösterilmektedir. Parantez içinde olmayan üstteki değerler yanal kayma değerleridir, pozitif olanlar sol yanaldır. Parantez içindeki değerler faya dik kayma değerleridir, pozitif olanlar yakınlaşmayı ifade eder. Hata elipsleri \%95 güven düzeyinde gösterilmiştir.

\section{CONCLUSIONS}

The strike of fault segments and the locations of pull-apart basins in northwest Anatolia indicate northwest - southeast compression and northeast southwest extension due to the right lateral strikeslip system which agrees with the majority of the focal mechanism solutions presented in this paper
(Figure 1b). Seismic activity on the western part of the southern branch is relatively higher than on the eastern part, however, the location of the Gölpazarı pull-apart basin and the re-evaluated MT cross section on the eastern side reveal the location of an alternative route for the southern branch (Figure 4). This new definition better 
explains the regional tectonics of the Gölpazarı pull-apart basin and provides a better fit of block modelling to GPS data. Slip distribution among the three branches of the NAFZ in the Marmara region based on GPS data demonstrate that the new route for the southern branch is the second most important branch in the region in terms of slip distribution along the NAFZ.
The suggested hypothesis for a new alternative southern route of the NAFZ, evidenced by morphotectonic features, seismology, GPS and recently published MT data, should be tested in the field by detailed structural analyses and seismic reflection studies.

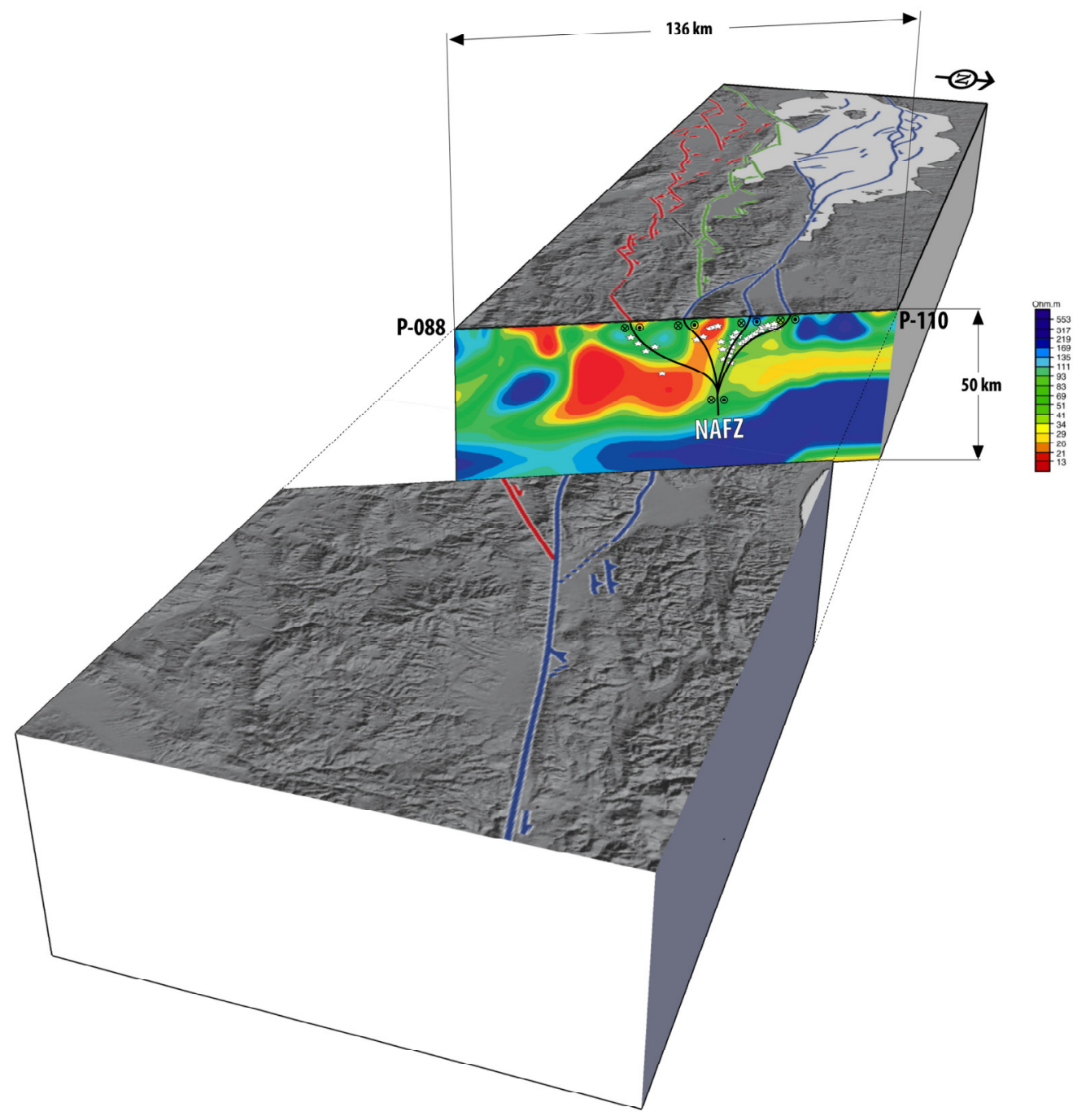

Figure 4. 3D perspective view of the three branched NAFZ in the Marmara region. The hypothesized southern branch is well correlated with the re-evaluated MT data of Kaya (2010).

Şekil 4. Marmara bölgesinde ü̧̧ kollu KAFZ'nun üç boyutlu perspektif görünümü. Güney kol hipotezi, Kaya (2010)'nun yeniden yorumlanmış MT verisi ile uyum içindedir. 


\section{GENIŞLETILMIŞ ÖZET}

Kuzey Anadolu Fay Zonu (KAFZ) doğuda dar bir makaslama zonu içinde yer alırken, batıda Bolu'dan itibaren geniş bir makaslama zonu içerisinde üç ana kola ayrlmaktadır (Şekil 1). Kuzey kol, Sapanca Gölü, İzmit Körfezi, Marmara Denizi ve Saros Körfezi'nden geçer. Orta kol Almacık bloğundan ayrlarak GeyvePamukova, İzik Gölü, Gemlik Körfezini izler ve Bandırma Körfezinden GB'ya döner. Güney kol'un KD ucunun Orta kol'dan Mekece üzerinden ayrllarak GB'ya doğru Yenişehir, Bursa, Ulubat Gölü güneyinden Mustafakemalpaşa'yı izleyerek Gönen civarında GB'ya döndüğ̈̈ bilinmektedir (Şekil 1) (Şengör, 1979; Barka ve Kadinsky-Cade, 1988; Barka ve Kuşçu, 1996; Emre vd., 1997; Armijo vd., 1999; 2002; Yaltırak, 2002; Gürer vd., 2003; 2006; Şengör vd., 2005; Kurtuluş ve Canbay, 2007; Y1lmaz ve Koral, 2007; Selim ve Tüysüz, 2013; Le Pichon vd., 2014).

Yakın zamanda yayınlanan Türkiye diri fay haritasında ise Güney kol, Bursa ve İnegöl arasında diri normal faylarla Eskişehir Fay Zonu'na bağlanır (Emre vd. 2011).

Yukarldaki güzergahlardan farklı olarak, Gölpazarı havzasının bir çek-ayır havza olarak değerlendirilmesinden sonra (Önde ve Gürbüz 2010; Gürbüz ve Seyitoğlu, 2014), MT verilerinin de yayinlanmasi (Kaya, 2010) ile, KAFZ'nun Güney kolu alternatif güzergahının daha doğuya Gölpazarı çek-ayır havzası ve Mudurnu üzerinden ana KAFZ'na Bolu'da bağlandĭ̆ı yönündeki bir hipotez bu makalede öne sürülmektedir.

Güney Marmara'nın genel yapısı KD doğrultulu săg yanal doğrultu atımlı faylar ile KB doğrultulu normal faylardan meydana gelmektedir. Bu yapılar büyük çek-ayır havza sistemlerinin elemanları olup, içlerinde Manyas ve Ulubat göllerini, Bursa, Yenişehir ve Gölpazarı ovalarını barındırmaktadır (Şekil 1 ve 2). KAFZ Güney kol'una ait alternatif güzergah ve ilişkili morfotektonik veriler Şekil 2'de sunulmuştur. Ayrıca Mudurnu fayı güzergahını belirlemek için Kaya (2010) tarafindan yayınlanan MT verisi yeniden yorumlanmıştır (Şekil 1 ve 2).

Önerilen Güney kol'un üzerindeki sismik etkinlik batıda doğuya göre daha fazladır. Sismik etkinlik verilerine yeniden konumlandirma işlemi yapılmış, ayrıca yeni odak mekanizma çözümleri elde edilmiştir (Şekil 1; Çizelge 1). Genel bölgesel gerilme dă̆llımı, săg yanal doğrultu atımlı sistemin çek-ayır doğası ile uyumlu gözükmektedir.

KAFZ'nun kollarl arasindaki kayma dağılımını incelemek için daha önceki fay tanımlamalarına uygun şekilde bir çalı̧̧ma yapılmış ve burada Güney kol'un en düşük kayma değerlerine sahip olduğu ve doğu segmentinin sol yanal kayma değerlerine sahip olduğu görülmüşü̈r (Şekil 3a). Marmara bölgesinde yeni önerilen Güney kol güzergahına uygun biçimde yapılan çalışmaya göre (Şekil 3b) güney blokta rezidüeller Şekil 3a'ya göre daha düşüktür ve yeni Güney kol ikinci en büyük kayma değerine sahiptir $(1.0-3.7 \mathrm{~mm} / \mathrm{yll})$.

KAFZ'nun Biga Yartmadast-GGD Marmara bölgesi anakarası üzerindeki güzergahı hakkındaki bu hipotez arazide daha detayl yapisal analizler ve sismik yansıma çalışmaları ile test edilmelidir.

\section{REFERENCES}

Aktuğ, B., Nocquet, J.M., Cingöz, A., Parsons, B., Erkan, Y., England, P., Lenk, O., Gürdal, M.A., Kılıçoğlu, A., Akdeniz, H., and Tekgül, A., 2009. Deformation of western Turkey from a combination of permanent and campaign GPS 
data: Limits to block-like behaviour. Journal of Geophysical Research, 114, B10404, doi: 10.1029/2008JB006000.

Aktuğ, B., Dikmen, Ü., Doğru, A., Özener, H., 2013a. Slip rates near Karlıova Triple Junction by GPS Observations. Journal of Geodynamics, 67, 21-29.

Aktuğ, B., Parmaksız, E., Kurt, M., Lenk, O., Kılıçoğlu, A., Gürdal, M.A., Özdemir, S., 2013b. Deformation of Central Anatolia: GPS Implications. Journal of Geodynamics, 67, 78-96.

Aktuğ, B., Doğru, A., Özener, H., Peyret, M., 2015. Slip rates and locking depth variation along central and easternmost segments of North Anatolian Fault. Geophysical Journal International, 202, 21332149.

Ambraseys, N.N., Jackson, J.A., 2000. Seismicity of the Sea of Marmara (Turkey) since 1500. Geophysical Journal International, 141, F1-F6.

Ambraseys, N., 2002. The seismic activity of the Marmara Sea Region over the last 2000 years. Bulletin of Seismological Society America, 92, $1-18$.

Ambraseys, N., 2009. Earthquakes in the Mediterranean and Middle East: a multidisciplinary study of seismicity up to 1900. Cambridge University Press.

Armijo, R., Meyer, B., Hubert, A., Barka, A., 1999. Westward propagation of the North Anatolian fault into the northern Aegean: Timing and kinematics. Geology, 27, 267-270.

Armijo, R., Meyer, B., Navarro, S., King, G., Barka, A., 2002. Asymmetric slip partitioning in the Sea of Marmara pull-apart: a clue to propagation processes of the North Anatolian Fault? Terra Nova, 14, 80-86.

Barka, A., Kadinsky-Cade, K., 1988. Strike-slip fault geometry in Turkey and its influence on earthquake activity. Tectonics, 7, 663-684.

Barka, A., Reilinger, R., 1997. Active tectonics of the Eastern Mediterranean region: deduced from
GPS, neotectonic and seismicity data. Annali di Geofisica, 40, 587-610.

Barka, A., 1992, The North Anatolian Fault Zone. Annales Tectonicae, 6, 164-195.

Barka, A.A., Kuşçu, İ., 1996, Extents of the North Anatolian fault in the İzmit, Gemlik and Bandırma bays. Turkish Journal of Marine Sciences, 2, 93106.

Çağlar, İ., İşseven, T., 2004. Two-dimensional geoelectrical structure of the Göynük geothermal area, northwest Anatolia, Turkey. Journal of Volcanology and Geothermal Research, 134, 183197.

Ekmekçi, N., Nazik, L., 2004. Evolution of GölpazarıHüyük karst system (Bilecik-Turkey): Indications of morpho-tectonic controls. International Journal of Speleology, 33, 49-64.

Ekström, G.A., England, P.C., 1989. Seismic strain rates in regions of distributed continental deformation. Journal of Geophysical Research, 94, 1023110257.

Emre, Ö., Erkal, T., Kazanc1, N., Görmüş, S., Görür, N., Kuşçu, İ., Keçer, M., 1997. Morphotectonics of the southern Marmara region during the Neogene and Quaternary: TÜBİTAK Project Report YDABÇAG-426/G, 36-68.

Emre, Ö., Doğan, A., Duman, T.Y., Özalp, S., 2011. Bursa (NK 35-12) quadrangle, active fault map series of Turkey: General Directorate of Mineral Research and Exploration, Ankara-Turkey, serial number 9 , scale $1 / 250,000,1$ sheet.

Eyidoğan, H., 1988. Rates of crustal deformation in western Turkey as deduced from major earthquakes. Tectonophysics, 148, 83-92.

Flerit, F., Armijo, R., King, G., Meyer, B., 2004. The mechanical interaction between the propagating North Anatolian Fault and the back-arc extension in the Aegean. Earth and Planetary Science Letters, 224, 347-362. 
Guidoboni, E., Comastri,A., Traina, G., 1994. Catalogue of Ancient Earthquakes in the Mediterranean area up to the 10th century. ING-SGA, Bologna 1: 504 p.

Gürbüz, A., 2010, Geometric characteristics of pullapart basins. Lithosphere, 2, 199-206.

Gürbüz, E., Seyitoğlu, G., 2014. Quaternary development of the Gölpazarı basin (NW Turkey). Geological Bulletin of Turkey, 57, 1-17.

Gürer, Ö.F., Kaymakçı, N., Çakır, Ş., Özburan, M. 2003. Neotectonics of the southeast Marmara region, NW Anatolia, Turkey. Journal of Asian Earth Sciences, 21, 1041-1051.

Gürer, Ö.F., Sangu, E., Özburan, M. 2006. Neotectonics of the SW Marmara region, NW Anatolia, Turkey. Geological Magazine, 143, 1-13.

Kaya, C., 2010. Deep crustal structure of northwestern part of Turkey. Tectonophysics, 489, 227-239.

Ketin, İ., 1948. Uber die tektonisch-mechanischen Folgerungen aus den grossen anatolischen Erdbeden des letzten Dozennimus. Geologische Rundschau, 36, 77-83.

Kondorskaya, N.V., Ulomov, V.I., 1999. Special catalogue of earthquakes of the Northern Eurasia from ancient times through 1995 (SECNE). Joint Institute of Physics of the Earth (JIPE), Russian Academy of Sciences, Moscow, Russia.

Koçyiğit A. 1988. Tectonic setting of the Geyve Basin: Age and total displacement of the Geyve Fault Zone. METU Journal of Pure and Applied Sciences, 21, 81-104.

Kurtuluş, C., Canbay, M.M., 2007. Tracing the middle strand of the North Anatolian Fault Zone through the southern Sea of Marmara based on seismic reflection studies. Geo-Marine Letters, 27, 27-40.

Le Pichon, X., Kreemer, C., 2010. The Mioceneto-present kinematic evolution of the eastern Mediterranean and Middle East and its implications for dynamics. Annual Review of Earth and Planetary Sciences, 38, 323-351.
Le Pichon, X., İmren, C., Rangin, C., Şengör, A.M.C., Siyako, M., 2014. The south Marmara Fault. International Journal of Earth Sciences, 103, 219231.

Matsu'ura, M., Jackson, D.D., and Cheng, A., 1986. Dislocation model for aseismic crustal deformation at Hollister, California. Journal of Geophysical Research, 91(B12), 2661-2674.

McCaffrey, R., 2002. Crustal block rotations and plate coupling, in Stein, S., and Freymueller, J.T., eds., Plate Boundary Zones American Geophysical Union Geodynamics Series 30, pp. 101-122. doi:10.1029/030GD06.

McCaffrey, R., 2005. Block kinematics of the Pacific-North America plate boundary in the southwestern United States from inversion of GPS, seismological, and geologic data. Journal of Geophysical Research, 110(B7), B07401, doi: 10.1029/2004JB003307.

McKenzie, D., 1972. Active tectonics of the Mediterranean region. Geophysical Journal of the Royal Astronomical Society, 30, 109-185.

Meade, J. B., Hager, B. H., McClusky, S.C., Reilinger, R.E., Ergintav, S., Lenk, O., Barka, A., Özener, H., 2002. Estimates of seismic potential in the Marmara sea region from block models of secular deformation constrained by Global Positioning System Measurments. Bulletin of the Seismological Society of America, 92, 208-215.

Nyst, M., Thatcher, W., 2004. New constrains on the active tectonic deformation of the Aegean. Journal of Geophysical Research, 109, B11406, doi: 10.1029/2003JB002830.

Okada, Y., 1985. Surface deformation due shear and tensile faults in a halfspace. Bulletin of the Seismological Society of America, 75, 11351154.

Önde, E., Gürbüz, A., 2010. Morphotectonic setting of the Gölpazarı pull-apart basin: Implications on the region between the North Anatolian and Eskişehir fault zones, NW Turkey. Geophysical Research Abstracts 12, EGU2010-944-2. 
Özener, H., Arpat, E., Ergintav, S., Doğru, A., Çakmak, R., Turgut, B., Doğan, U., 2010. Kinematics of the eastern part of the North Anatolian Fault Zone. Journal of Geodynamics, 49, 141-150.

Papazachos, B., Kiratzi, A., Papadimitriou, E., 1991. Regional focal mechanisms for earthquakes in the Aegean Area. Pure and Applied Geophysics, 136, 407-420.

Reilinger, R., McClusky, S., Vernant, P., Lawrence, S., Ergintav, S., Çakmak, R., Özener, H., Kadirov, F., Guliev, I., Stepanyan, R., Nadariya, M., Hahubia, G., Mahmoud, S., Sakr, K., ArRajehi, A., Paradissis, D., Al-Aydrus, A., Prilepin, M., Guseva, T., Evren, E., Dmitrotsa, A., Filikov, S.V., Gomez, F., Al-Ghazzi, R. and Karam, G., 2006. GPS constraints on continental deformation in the Africa - Arabia-Eurasia continental collision zone and implications for the dynamics of plate interactions. Journal of Geophysical Research, 111, B05411, doi: 10.1029/2005JB004051.

Selim, H.H., Tüysüz, O., 2013. The Bursa-Gönen depression, NW Turkey: a complex basin developed on the North Anatolian Fault. Geological Magazine, 150, 801-821.

Seymen, İ. 1975. Kelkit Vadisi Kesiminde Kuzey Anadolu Fay Zonunun Tektonik Özelliği: Dr. Eng., İstanbul Tek. Univ., Maden Fak., 192 pp.

Soysal, H., Sipahioğlu, S., Koçak, D., Altınok, Y., 1981. A historical earthquake catalogue for Turkey and its surrounding-2100 BC to 1900: TÜBİTAK Project Report TBAG-341.

Şengör, A.M.C., 1979. The North Anatolian transform fault: its age, offset and tectonic significance. Journal of the Geological Society London, 136, 269-282.

Şengör, A.M.C., Tüysüz, O., İmren, C., Sakınç, M., Eyidoğan, H., Görür, N., Le Pichon, X., and Rangin, C., 2005. The North Anatolian Fault: A New Look: Annual Review of Earth and Planetary Sciences, doi: 10.1146/annurev. earth.32.101802.120415.

Tan, O., Tapırdamaz, M.C., and Yörük, A., 2008. The earthquake catalogues for Turkey. Turkish Journal of Earth Sciences, 17, 405-418.

Tatar, O., Poyraz, F., Gürsoy H., Çakır, Z., Ergintav, S. Akpınar, Z., Koçbulut, F., Sezen, F., Türk, T., Hastaoğlu, K.Ö., Polat, A., Mesci, B.L., Gürsoy, Ö., Ayazlı İ.E., Çakmak, R., Belgen, A Yavaşoğlu, A., 2012, Crustal deformation and kinematics of the Eastern Part of the North Anatolian Zone (Turkey) from GPS measurements. Tectonophysics, 518$521,55-62$

Yaltırak, C., 2002. Tectonic evolution of the Marmara Sea and its surroundings. Marine Geology, 190, 493-529.

Yavaşoğlu, H., Tarı, E., Tüysüz, O., Çakır, Z., Ergintav, S., 2011. Determining and modelling tectonic movements along the central part of the North Anatolian Fault (Turkey) using geodetic measurements. Journal of Geodynamics, 51, 339 343.

Y1lmaz, M., Koral, H., 2007. Neotectonic features and geological development of the Yenişehir basin (Bursa). İstanbul Yerbilimleri Dergisi 20, 21-32.

$\begin{array}{ll}\text { Makale Geliş Tarihi } & : 4 \text { Şubat } 2016 \\ \text { Kabul Tarihi } & : 18 \text { Mart } 2016 \\ \text { Received } & : 4 \text { February } 2016 \\ \text { Accepted } & : 18 \text { March } 2016\end{array}$

